\title{
Langmuir Films of Normal-Alkanes on the Surface of Liquid Mercury
}

\section{Citation}

Kraack, H., B. M. Ocko, Peter S. Pershan, E. Sloutskin, and M. Deutsch. 2003. Langmuir films of normal-alkanes on the surface of liquid mercury. Journal of Chemical Physics 119(19): 10339-10349.

\section{Published Version}

doi:10.1063/1.1618211

\section{Permanent link}

http://nrs.harvard.edu/urn-3:HUL.InstRepos:10354436

\section{Terms of Use}

This article was downloaded from Harvard University's DASH repository, and is made available under the terms and conditions applicable to Other Posted Material, as set forth at http:// nrs.harvard.edu/urn-3:HUL.InstRepos:dash.current.terms-of-use\#LAA

\section{Share Your Story}

The Harvard community has made this article openly available.

Please share how this access benefits you. Submit a story.

\section{Accessibility}




\title{
Langmuir films of normal-alkanes on the surface of liquid mercury
}

\author{
H. Kraack \\ Department of Physics, Bar-Ilan University, Ramat-Gan 52900, Israel \\ B. M. Ocko \\ Department of Physics, Brookhaven National Laboratory, Upton, New York 11973 \\ P. S. Pershan \\ Department of Physics, Harvard University, Cambridge, Massachusetts 02138 \\ E. Sloutskin and M. Deutsch ${ }^{\text {a) }}$ \\ Department of Physics, Bar-Ilan University, Ramat-Gan 52900, Israel
}

(Received 30 June 2003; accepted 20 August 2003)

\begin{abstract}
The coverage dependent phase behavior of molecular films of $n$-alkanes $\left(\mathrm{CH}_{3} \mathrm{CH}_{n-2} \mathrm{CH}_{3}\right.$, denote $\mathrm{Cn}$ ) on mercury was studied for lengths $10 \leqslant n \leqslant 50$, using surface tensiometry and surface x-ray diffraction methods. In contrast with Langmuir films on water, where roughly surface-normal molecular orientation is invariably found, alkanes on mercury are always oriented surface-parallel, and show no long-range in-plane order at any surface pressure. At a low coverage a two-dimensional gas phase is found, followed, upon increasing the coverage, by a single condensed layer ( $n$ $\leqslant 18$ ), a sequence of single and double layers $(19 \leqslant n \leqslant 20 ; n \geqslant 26)$, or a sequence of single, double, and triple layers $(22 \leqslant n \leqslant 24)$. The thermodynamical and structural properties of these layers, as determined from the measurements, are discussed. () 2003 American Institute of Physics.
\end{abstract}

[DOI: $10.1063 / 1.1618211]$

\section{INTRODUCTION}

Langmuir films have traditionally referred to quasi-twodimensional (2D) monomolecular films of amphiphiles residing on the surface of water, with their hydrophilic headgroups inside, and their hydrophobic tails outside the water subphase. Although similar films are already mentioned by the ancient Greek and Chinese sailors, the modern study of these films started with experiments carried out by Agnes Pockels at her home over a century ago. ${ }^{1} \mathrm{~A}$ few years later Lord Rayleigh realized that these films had a thickness of only a single molecule. ${ }^{2}$ Since these first studies Langmuir films have been studied extensively for more than a century by physicists, as a model for two-dimensional matter, ${ }^{3}$ by biologists as a model for the cell membrane, ${ }^{4}$ by chemists as a template for oriented growth of crystals from solution, ${ }^{5}$ etc. Recently, Langmuir films have been studied for their potential use in nanoscale control of matter: nanoengineering in general and nanopatterning of surfaces in particular. ${ }^{6}$ They are also being widely investigated for molecular electronics applications, ${ }^{3,7}$ and for studying the formation of supramolecular structures. ${ }^{8,9}$

For most of the 20th century, Langmuir films were studied extensively by a variety of macroscopic methods, most prominently by surface tensiometry which provides surface pressure-molecular area isotherms. ${ }^{10}$ However, direct structure determination of Langmuir films by $\mathrm{x}$-ray techniques with $\AA$ resolution became possible only two decades ago, when synchrotron-based liquid surface $\mathrm{x}$-ray reflectometry and grazing incidence diffraction methods were

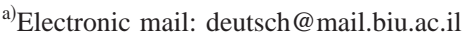

developed, ${ }^{11-14}$ and $\AA$-resolution measurement of the surface structure of water was published. ${ }^{15}$ Following the first x-ray structural studies of water-supported Langmuir monolayers in $1987,{ }^{16}$ these methods have been extensively employed to investigate the structure of a wide range of mono and multicomponent Langmuir monolayers under a broad spectrum of chemical and physical conditions., ${ }^{3,13,14,17,18}$ One of the crowning achievements of this activity was the complete determination of the temperature-surface pressure phase diagram of Langmuir films of fatty acids on water, demonstrating its universality upon chain length variation. ${ }^{19}$ A detailed theoretical description and interpretation of this phase diagram within a Landau-type mean-field theory was also achieved. $^{3}$

A rich array of interactions is possible in a liquidsupported Langmuir film of amphiphilic molecules. For example, for simple fatty acid molecules at the surface of an aqueous salt solution the chains interact via van der Waals forces, the headgroup may have competing interactions of hydrogen bonding with the water, and screened Coulomb interaction with the hydrated, or bare, ions in the subphase, etc. However, the dominant interaction, invariably present for organic monolayers on aqueous subphases, is the strong hydrophobic repulsion of the amphiphile's hydrocarbon tail from the aqueous subphase. The hydrophylic attraction of the headgroup in combination with the hydrophobic repulsion of the chains and the chain-chain attraction, tend to orient the amphiphilic molecules in the condensed phases of Langmuir films on water roughly normal to the surface. Since until very recently all subphases employed for $\mathrm{x}$-ray studies of Langmuir films were aqueous, the molecular orientation was invariably found to be along, or slightly tilted from, the sur- 
face normal. To the best of our knowledge, the only nonaqueous subphase employed in x-ray studies of a Langmuir film is formamide, ${ }^{17,20}$ which is also polar and induces the same surface-normal molecular orientation.

To investigate the role of the molecules' interaction with the subphase in the determination of the Langmuir film's structure we have studied the structure of Langmuir films of organic molecules on mercury. ${ }^{21}$ Here the interaction of the chains with the subphase is attractive, rather than repulsive, and the hydrophobicity of the molecular tail plays no role. In this case, a surface-parallel molecular orientation could be expected, at least for a range of coverages. Such orientations were not observed in any organic Langmuir monolayer on water, although high tilts from the surface normal were detected in crystallites of alkanes $(n \sim 24)$ formed on water. These crystallites self-assemble spontaneously at zero surface pressure from excess material placed on water for 20 $<n<30 .{ }^{17,22}$ Mercury as a subphase for Langmuir films offers several additional advantages. The high surface energy of mercury, $500 \mathrm{~mJ} / \mathrm{m}^{2}$, as compared to that of water, $72 \mathrm{~mJ} / \mathrm{m}^{2}$, should enhance the spreading of surface films, and induce spreading in compounds not readily, or not at all, spreadable on water. Since the solubilities of all organic compounds, and most inorganic ones, in mercury are practically zero, compounds which can not be studied as Langmuir films on water due to their high water solubility could still be investigated on mercury. Also, the higher surface tension of mercury allows the extension of the x-ray reflectivity measurements to fourfold larger scattering vectors than those achievable on water, yielding a commensurately higher resolution. The first atomic resolution measurement of the surface structure of pure mercury, a prerequisite for any mercury-supported Langmuir film structure determination, was published only recently. ${ }^{23}$ Following our studies of dense self-assembled alkyl-thiol monolayers ${ }^{24}$ and multilayers ${ }^{25}$ and, very recently, of a Langmuir film of stearic fatty acid $^{21}$ on mercury, we present here a study of the structure of Langmuir films of normal-alkanes on mercury as a function of surface coverage, for molecular length of $10 \leqslant n$ $\leqslant 50$. Surface tensiometry was used to measure surface pressure-molecular area isotherms, and synchrotron-based surface scattering $\mathrm{x}$-ray methods were employed to determine the structure at various points along the isotherm.

Finally, we note that self-assembled mono- and multilayers on solid substrates, mostly alkyl-thiols on gold, have been also investigated extensively by $\mathrm{x}$-ray, and other, methods. ${ }^{26}$ Surface-parallel molecular orientations of such self-assembled monolayers (SAMs) were found at certain coverage ranges. However, these SAMs should be clearly distinguished from Langmuir films on a liquid substrate. Obviously, on a liquid subphase the film's molecules are at least as mobile as those of the subphase, while on a solid one the film's molecules are bound (more or less strongly) to static sites on the surface of the substrate. Thus, varying the coverage is considerably simpler, the surface coverage is more uniform and more likely to be a true equilibrium on a liquid surface than on a solid one. More importantly, in the most extensively studied SAMs by far, alkyl-thiols on gold, the crystalline structure of the gold surface was found to deter- mine the SAM's structure by epitaxy, both in the surface-normal ${ }^{27}$ and the surface-parallel phases. ${ }^{28} \mathrm{An}$ epitaxial arrangement was also found for alkane SAMs on graphite, ${ }^{29}$ on single-crystals of copper, ${ }^{30}$ and gold. ${ }^{31}$ This epitaxy of the organic monolayer to the structure of the subphase does not exist, of course, in the case of mercury, where the liquid surface of the subphase does not possess longrange order, and hence can not force the Langmuir film to conform to the order dictated by the surface's corrugation potential, as is the case in SAMs. ${ }^{26}$

\section{EXPERIMENT}

\section{A. The trough}

A specially designed Langmuir trough, suitable for simultaneous surface tension and $\mathrm{x}$-ray studies, was used. The trough itself is milled from KelF and has inner dimensions of $175 \times 65 \times 3.5 \mathrm{~mm}^{3}$, with a thin $(0.3 \mathrm{~mm})$ bottom to allow good thermal contact with the fixed-temperature plate on which the trough is mounted. The temperature of the plate can be controlled to $\pm 0.2{ }^{\circ} \mathrm{C}$ by water circulation from a commercial water bath/circulator system. The trough is enclosed in an hermetically sealed aluminum box, equipped for a flow of pure helium or nitrogen gas to minimize surface contamination and oxidation of the mercury. The enclosure has thin Kapton entrance and exit windows for the $\mathrm{x}$ rays. The mercury is fed into the trough from the center of a stainless steel reservoir mounted on the top plate of the enclosure, through a valve and a fine capillary extending through the plate almost to the trough's bottom. Since oxides and organic contaminants float to the surface of the mercury in the reservoir, this arrangement allows the introduction of clean mercury into the trough without breaking the seal of the enclosure, and without exposing the mercury to air.

\section{B. The surface tension balance}

The surface tension is measured using the Wilhelmy plate method. We use a platinum plate $(12.5 \times 10$ $\times 0.5 \mathrm{~mm}^{3}$ ). To obtain good wetting of the plate by the mercury it must be first amalgamated with mercury by placing the clean plate into a mercury bath in a glass dish, which is then placed in a sealed aluminum cell under a nitrogen atmosphere. The cell is then heated to $T=125^{\circ} \mathrm{C}$ and left at this temperature for $48 \mathrm{~h}$. This procedure ${ }^{32}$ produces a thin amalgam layer on the plate, which is stable for periods up to 2 months. Each time a new mercury subphase is introduced into the trough, the plate is removed from the balance, and rubbed lightly with tissue paper. This renews the amalgamation layer. The plate is then "washed" several times by dipping into clean mercury to remove contaminants, and is then ready to use.

The balance consists of a leaf spring, which carries the platinum plate, and a linear variable differential transformer (LVDT). The LVDT produces an output voltage proportional to the displacement of the spring upon immersion of the plate's edge into the mercury. The displacement is proportional to the force exerted on the leaf spring by the surface tension $\sigma$ pulling on the plate. Repeated readings of $\sigma$ in an unperturbed system vary by less than $\Delta \sigma=0.05 \mathrm{mN} / \mathrm{m}$. 


\section{Materials}

Mercury was purchased from Merck Co. (triple distilled, 99.999\% pure) and Bethlehem Apparatus Co. (quadrupple distilled $99.99995 \%$ pure). Alkanes were purchased from Aldrich and were at least $98 \%$ pure. All materials were used as received without further purification. Measurements on the same alkane with different purities $(99.5 \%, 98 \%)$ did not show any differences in the surface pressure-molecular area isotherms. Stock solutions were prepared with molarities in the range of $3-8 \times 10^{-4}$ using HPLC grade, $99.9 \%$ pure chloroform. Film deposition was done by a micropipette, through a sealable hole in the enclosure's top plate.

\section{Measurement methods}

\section{Surface pressure-molecular area isotherms}

The surface pressure, $\pi=\sigma_{0}-\sigma$, is defined as the difference between the surface tension of bare $\left(\sigma_{0}\right)$ and filmcovered $(\sigma)$ mercury. $\pi$ varies with the surface coverage, given by the area per molecule $A$, through the dependence of $\sigma$ on $A .^{10}$

To measure an isotherm, i.e., a $\pi$ versus $A$ curve, the trough was first cleaned thoroughly with isopropanol and chloroform, the enclosure sealed, and flushed for one hour with a flow of (99.999\% pure) nitrogen, when only isotherm measurements are planned, or (99.999\% pure) helium, when $\mathrm{x}$-ray measurements are planned. The trough was then filled with mercury from the reservoir through the capillary to a level of about $1 \mathrm{~mm}$ above its rim. The measured surface tension of freshly filled mercury varied between $\sigma_{0}$ $=475 \mathrm{mN} / \mathrm{m}$ and $\sigma_{0}=495 \mathrm{mN} / \mathrm{m}$, depending on the quality of the amalgam layer on the Wilhelmy plate and its immersion depth into the mercury. Film deposition began immediately after filling the trough, to minimize the collection of impurities on the bare mercury surface. Two reasons render the coverage control by a travelling barrier, as done for water, less advantageous here. First, well-sealing barriers are notoriously more difficult to construct for mercury than for water. ${ }^{33}$ Second, the large $A$-range of interest here entails a high compression ratio of 20-25. This, in turn, requires a prohibitively long trough and long traveling range for the barrier. Thus we measured the isotherms by consecutive deposition of accurately measured volumes of the stock solution by a micropipette, adding from 1 to 10 microliters of solution at each step. Waiting time following each deposition varied from about half a minute at low coverage to $30 \mathrm{~min}$ at high coverage, and strongly depended upon the chain length of the alkane, as we discuss below. The required waiting time was determined by monitoring the variation of $\pi$ from the moment of deposition until no further change is observed in $\pi$.

A typical trace of the surface pressure vs time for a long (C22) chain alkane is shown in Fig. 1. When solution is added, a jump in $\pi$ is observed, followed by a relaxation period to a new, higher value of $\pi$. For C22 the relaxation time was found to be almost independent of $A$ and $\pi$. Equilibrium in $\pi$ is reached within less than a minute after each deposition, indicating a very good spreading of the alkane molecules on the mercury surface. The inset to Fig. 1 shows

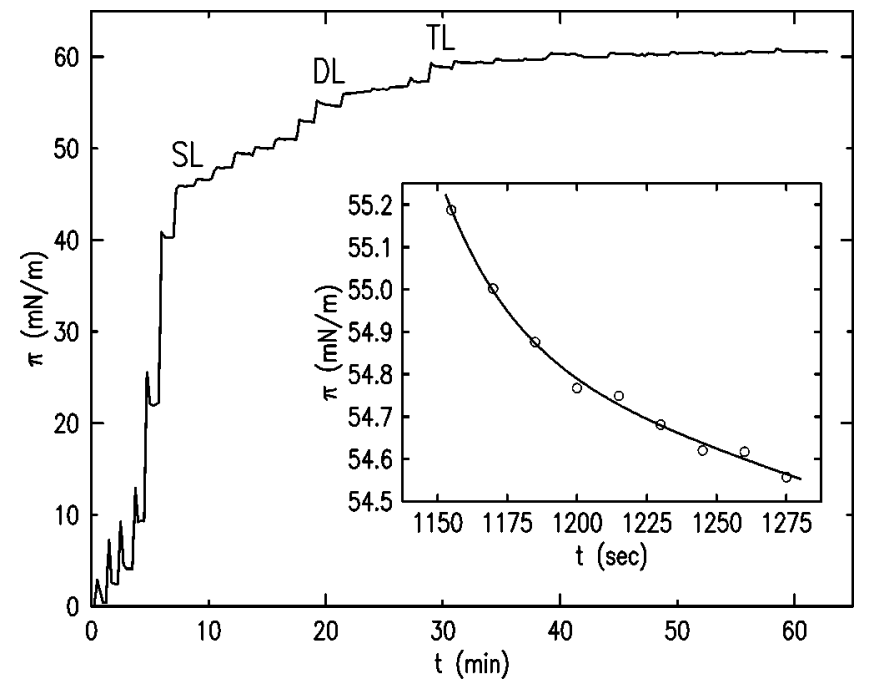

FIG. 1. The time dependence of the surface pressure $\pi$ during the stepwise deposition of a Langmuir film of C22. SL, DL, and TL indicate the formation of single, double, and triple layers of molecules. The inset is an expanded time scale plot of the measured (open circles) and fitted (solid line) exponential relaxation of $\pi$ near the onset of the DL phase. The fitted relaxation time constant here is $\sim 50 \mathrm{~s}$.

an example of the measured relaxation of $\pi$ (open circles) following a deposition step at $t \approx 1150 \mathrm{~s}$. The surface pressure is observed to relax exponentially, with a time constant of $50 \mathrm{~s}$ (solid line). Figure 1 shows three steps in $\pi$. These will be shown below to be the formation of single (SL), double (DL), and triple (TL) layers of flat-lying molecules.

\section{X-ray measurements}

The molecular structure of the Langmuir films was studied at various coverages using surface-specific x-ray techniques. The $\mathrm{X}$-ray measurements were carried out at the Harvard/BNL liquid surface spectrometer at beamline X22B, NSLS, Brookhaven National Laboratory, at wavelengths of $\lambda=1.55-1.58 \AA$. The trough was supported on an active vibration isolation unit, mounted on the spectrometer. This arrangement was demonstrated in previous measurements ${ }^{23,24}$ to eliminate vibrational pickup from the environment, the dominant effect which severely curtailed the measurement range in all early studies of the surface structure of mercury. ${ }^{34}$

A detailed description of the x-ray measurement methods used is available in the literature ${ }^{13,35}$ and will not be repeated here. We have carried out x-ray reflectivity (XR) measurements, which yield information on the surfacenormal structure of the Langmuir film such as its surfacenormal density profile and its surface roughness. The inplane order was investigated by grazing incidence diffraction (GID). The XR measurements employed a point detector while the GID was measured using a linear position sensitive detector, which allows a simultaneous measurement of a full Bragg rod (BR) when GID peaks are observed. To minimize beam damage, sample exposure times were kept to a minimum by using an automatic shutter upstream of the trough. This was opened only for counting, and kept closed during spectrometer movements. 


\section{X-ray reflectivity modeling}

$\mathrm{X}$-ray reflectivity, $R\left(q_{z}\right)$, is measured as a function of the scattering vector $q_{z}=(4 \pi / \lambda) \sin (\alpha)$; where $\alpha$ is the grazing angle of incidence of the $\mathrm{x}$ rays on the liquid surface. The measured XR is often presented normalized by the XR of an ideally flat and abrupt interface, the so-called Fresnel reflectivity $R_{F}\left(q_{z}\right){ }^{35}$ The density profile of the alkane-covered mercury was modeled by a box model of a single box for each alkane layer and 6 boxes for the mercury. For the bare mercury surface 8 slabs were used. Following a number of trial fits varying various fit parameters in different combinations, a final model was adapted allowing us to obtain good fits to all measured curves, with reasonable fit parameters. The final model was as follows.

For the mercury a fixed box width of $1.3 \AA$ and a fixed interfacial roughness of $0.7 \AA$ was used for all 6 boxes representing the near-surface oscillatory density profile of the mercury. Thus, only the height (i.e., density) of each box was varied, except that the density of the first box was kept fixed at $5.5 \mathrm{e} / \AA^{3}$. A separate interfacial roughness parameter was assigned to the mercury-alkane interface, and varied in the fit. The mercury's bulk electron density was kept fixed at the $\rho_{e}=3.25 \mathrm{e} / \AA^{3}$ calculated from the known mass density of mercury. This model is admittedly more restricted and less detailed than that used previously in the studies of the surface structure of bare mercury. ${ }^{23}$ However, the $q_{z}$-range of the present measurements is restricted to $q_{z} \lesssim 1.5-1.7 \AA^{-1}$ by the need to minimize beam damage to the alkane film. This range excludes almost all of the quasi-Bragg peak originating in the mercury layering at the surface, which peaks at $\sim 2.2 \AA^{-1}$ and extends out to $\sim 2.5 \AA^{-1}$. Only the beginning of the rise of the layering peak can be observed at the high- $q_{z}$ end of the $q_{z}$-range in our measurements [see Fig. 3(a) below]. Thus, the measured $R\left(q_{z}\right)$ in this study are not sensitive to, and cannot support a full modeling of the mercury layering. Nevertheless, the variation of the alkanemercury interface, which is rougher for lower coverage than for higher one, still requires some flexibility in the modeling of the mercury surface. A comparison of the mercury density profiles obtained here employing our restricted model with those obtained in our previously-published detailed studies of bare mercury ${ }^{23}$ shows that the present simple model captures the main features of the layering, though obviously not all the details, particularly in the mercury layers lying below the first two surface-adjacent layers. As the results presented below show, the model described here for the mercury is flexible enough to provide a good fit to the measured $R\left(q_{z}\right)$ of the alkane-covered mercury surface with physically reasonable parameter values, while keeping the number of fit parameters reasonably small.

For the alkane layers a fixed density of $\rho_{e}=0.3 e / \AA^{3}$ was employed, and the width and roughness parameters were varied in the fit. Using a fixed $\rho_{e}$ deviating by up to $\pm 5 \%$ from this value yields equally good fits. In the coexistence regimes (single/double and double/triple layers) all layers except the top layer are assumed to have a $100 \%$ coverage, and are represented by a box having a fixed density of $0.3 e / \AA^{3}$. The top, incomplete layer was represented by a box having the fixed width of a completed layer, and its density $\rho_{e}$ and

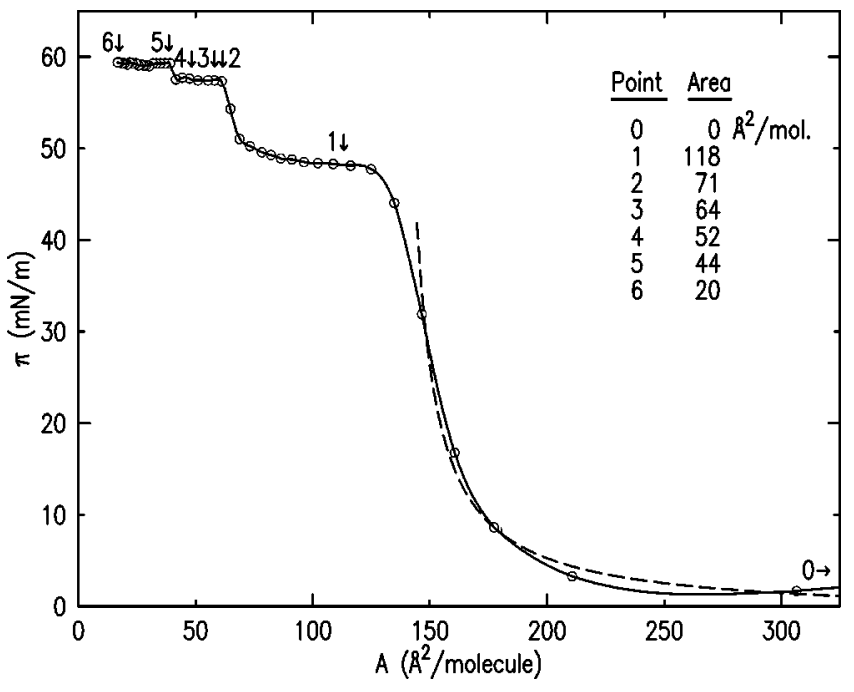

FIG. 2. Surface pressure $(\pi)$-molecular area $(A)$ isotherm for docosane (C22) on mercury (solid line+ points). The dashed line is a fit to the Volmer equation for a gas of flat-lying molecules having hard-core interactions. The three abrupt $\pi$ changes at $\pi \approx 138,64$, and $41 \AA^{2} /$ molecule suggest the consecutive formation of a single, double, and triple layers of flat-lying molecules.

roughness allowed to vary in the fit. The density obtained from the fit, $\rho^{\prime}$, was then used to derive the coverage fraction of the top layer as $F=\rho^{\prime} / 0.3$. This procedure is equivalent to a coherent averaging over the coexisting differentphase domains, an assumption supported by the good contrast observed in the undulations in $R / R_{F},{ }^{36}$ Almost identical $R$ curves were obtained by using the values of the fitderived parameters and assuming incoherent averaging over the domains (i.e., summing reflected intensities rather than amplitudes).

Finally, all fits were done using the matrix method implementation ${ }^{37}$ of the Parratt formalism. ${ }^{38}$ As the results discussed below indicate, the model and strategy employed here result in good fits and a consistent variation of the fitted parameter values with coverage, keeping, at the same time, the number of fit parameters down to a manageable number, and the interparameter correlation to a minimum.

\section{RESULTS AND DISCUSSION}

We first discuss in detail the results obtained for docosane (C22), and then extend the discussion to alkanes of other chain lengths.

\section{A. Docosane}

\section{Surface pressure-molecular area isotherms}

The measured $\pi-A$ isotherm of $\mathrm{C} 22$, taken at $T=23{ }^{\circ} \mathrm{C}$ is shown in Fig. 2. The measured points are shown as open circles and the solid line is a smoothed curve connecting the points. Comparing this isotherm with typical isotherms on water, ${ }^{10}$ two differences stand out immediately in the low- $A$ region. One is the high surface pressure obtained, $\pi$ $\approx 60 \mathrm{mN} / \mathrm{m}$, which is much higher then the collapse pressures observed in Langmuir films on aqueous subphases. ${ }^{10}$ Similarly high collapse pressures were found for other organic molecules on mercury. ${ }^{21,39,40}$ The fact that the collapse 
pressure on $\mathrm{Hg}$ is much higher than that on water may point towards film buckling as a possible collapse mechanism here. For such a mechanism the attraction of the monolayer to the surface is the dominant factor in determining the collapse pressure. Thus, one would expect a much higher collapse pressure for the strongly-attracting mercury than for water, where the chains are repelled from the surface, and the relatively weak hydrogen bond of the headgroup to the water anchors the molecules to the surface. While this expectation indeed agrees with the observation of a lower collapse pressure on water and a higher one on $\mathrm{Hg}$, more direct experimental evidence is required for a definite conclusion on the nature of the collapse mechanism prevailing here.

The second outstanding feature in Fig. 2 is the absence of a fast increase in $\pi$ upon reducing $A$ at the low- $A$ end of the isotherm. Such an increase is a common feature of all isotherms measured to date for amphiphiles on aqueous subphases, where it manifests the existence of a condensed solid phase of low compressibility. ${ }^{3,17}$ The absence of this feature here is discussed below, based on the x-ray measurements in this $A$-region.

As Fig. 2 shows, reducing the molecular area $A$ from $\sim 350 \AA^{2} /$ molecule to $\sim 200 \AA^{2} /$ molecule causes only a very small change in the surface pressure, as expected for a dilute 2D gas of molecules. However, a further reduction below $200 \AA^{2} /$ molecule produces a very steep rise from $\pi$ $=2 \mathrm{mN} / \mathrm{m}$ at $\sim 250 \AA^{2} /$ molecule to $\pi=47 \mathrm{mN} / \mathrm{m}$ at $135 \AA^{2} /$ molecule. Similar to our previously measured isotherm of stearic acid on mercury, ${ }^{21}$ the isotherm here can be reasonably well fit in this region by the Volmer equation $\pi\left(A-A_{1}\right)=k T$, which describes a two-dimensional hardcore-interacting gas of molecules. The exclusion area $A_{1}$ is due to the finite size of the molecules. The fit is shown in a dashed line in Fig. 2, and yields an exclusion area $A_{1}$ $=138 \AA^{2} /$ molecule. This value is very close to the area of a C22 molecule lying flat on the mercury surface: $A=l \times w$ $=29 \times 4.8 \AA^{2}=139 \AA^{2}$. Thus, the onset of the first plateau in the isotherm in Fig. 2 can be concluded to mark the completion of a densely-packed single layer (SL) of flatlying molecules. Note that although the two-dimensional version of the van der Waals equation of state predicts an exclusion area of twice the molecular area, ${ }^{41}$ this result is derived using an expansion valid in the low-concentration limit, i.e., when $A \gg A_{1}$ in our case. When the molecules are packed densely on the surface, i.e., when $A \rightarrow A_{1}$ in our case, a number of theoretical ${ }^{42,43}$ and experimental studies on Langmuir films on water, ${ }^{44}$ show that the exclusion area is very close to the area taken up by a single molecule.

When $A$ is reduced below $A_{1}$, a series of nearly flat plateaus are observed at $\pi(A) \approx 47,56$, and $58 \mathrm{mN} / \mathrm{m}$. The transitions between plateaus are abrupt, and occur at $A=64$ and $41 \AA^{2} /$ molecule, respectively. The fact that these transitions occur at $A$ values which are roughly one-half and onethird of the molecular area of a single flat-lying molecule strongly suggests that double and triple layers of flat-lying molecules form at these $A$ values. To confirm the suggested stepwise growth of the three layers, obtain their thicknesses, and detect possible in-plane order within these layers, $x$-ray measurements were carried out at the points marked by num-
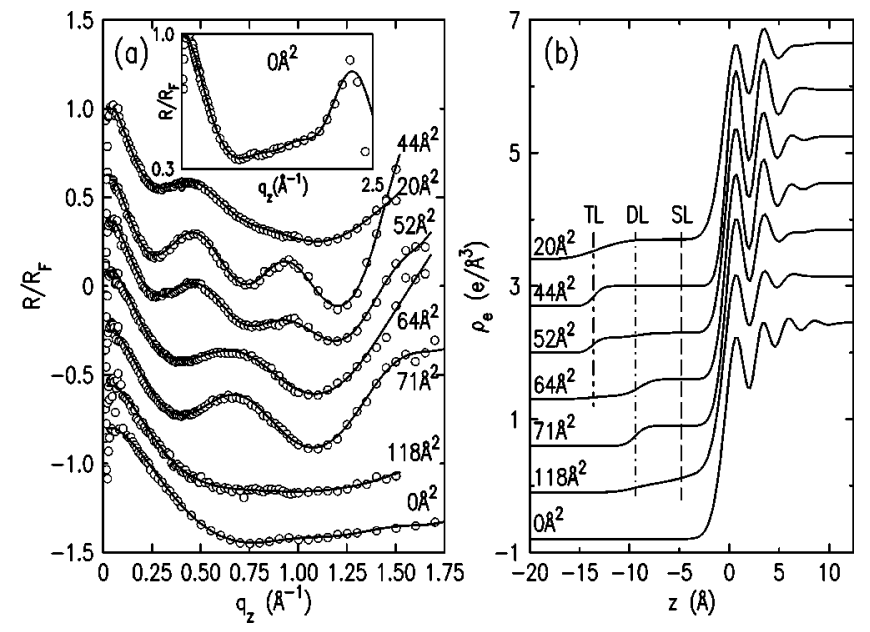

FIG. 3. (a) Measured Fresnel-normalized x-ray reflectivity curves, $R\left(q_{z}\right) / R_{F}\left(q_{z}\right)$, (open circles) of docosane (c22) on mercury, with their boxmodel fits (lines). Curves are shifted vertically by $\sim 0.3$ each for clarity. Inset: Same for the bare mercury surface. (b) The model density profiles obtained from the fits in (a). The successive formation of three layers of flat lying molecules, marked by the vertical lines for single (SL), double (DL), and triple (TL) layers, is clearly observed.

bers on the isotherm in Fig. 2. The results of these measurements are detailed in the next section.

\section{X-ray reflectivity}

The measured Fresnel-normalized $\mathrm{XR}, R / R_{F}$, of $\mathrm{C} 22$ is shown in Fig. 3(a) (open circles) as a function of coverage, along with their box-model fits (solid lines). The density profiles derived from the fit are shown in Fig. 3(b), with $z=0$ taken at the position of the mercury-alkane interface, and the positive $z$-axis pointing into the subphase. The previously-detected surface layering of mercury ${ }^{23}$ is indicated by the peak at $q_{z} \approx 2.2 \AA^{-1}$ of the bare mercury reflectivity curve in the inset of Fig. 3(a). It is also implied by the rise at the high- $q_{z}$ end of the other (smaller-range) reflectivity curves shown in Fig. 3(a). The corresponding layering is shown in the real-space density profiles in Fig. 3(b).

The fits yield a surface roughness in the range of 1.1 $\pm 0.3 \AA$, close to that of a pure mercury surface. The fit for $A=118 \AA^{2} /$ molecule, slightly lower than the area of a lyingdown molecule $\left(A_{1}=139 \AA^{2}\right)$, yields an alkane layer thickness of $4.7 \AA$, with a $100 \%$ complete first layer and a $40 \%$ complete second layer. The fits for $A=71,64,52$, and $44 \AA^{2} /$ molecule, at the onset, about one third along, and near the end of the second plateau and at the onset of the third plateau, yield, respectively, a $100 \%$ complete double layer $9.4 \AA$ thick, a $100 \%$ complete double layer with a $20 \%$, $70 \%$, and $100 \%$ complete third layer, respectively. The triple layer is found to be $13.5 \AA$ thick. When the coverage is increased further towards $20 \AA^{2} /$ molecule no further increase is detected in the number of layers either by $\mathrm{x}$-ray or isotherm measurements. The only observable effect in the $\mathrm{XR}$ measurements is a strong increase in the alkane-air interfacial roughness, from $\sim 1.0 \AA$ for a triple layer at $\mathbf{5}$ (44 $\AA^{2} /$ molecules), to $2.3 \AA$ for the highest measured coverage at $6\left(20 \AA^{2} /\right.$ molecules $)$. This high roughness suppresses all but the first oscillation in the XR curve. The layer 


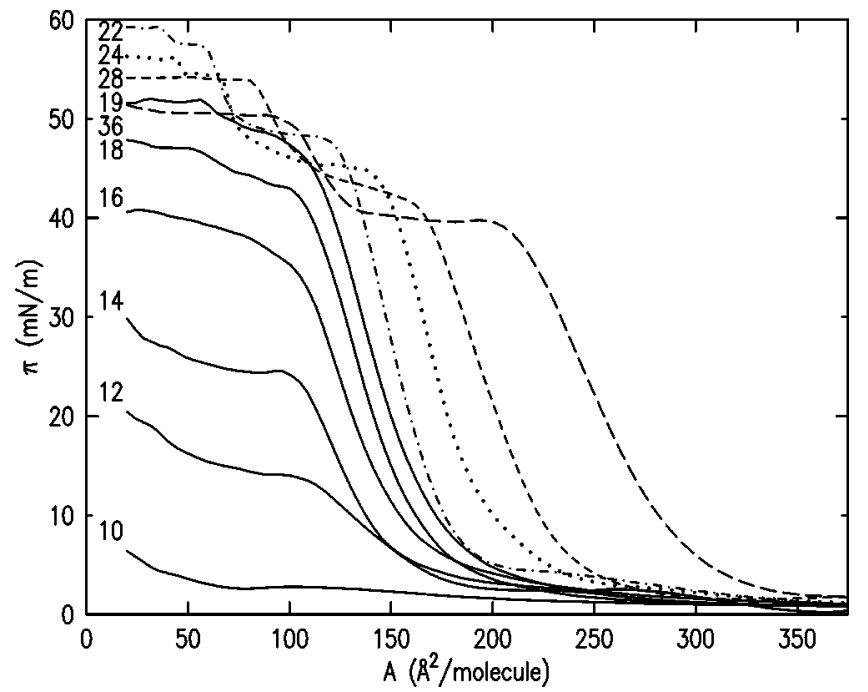

FIG. 4. Measured $\pi-A$ isotherms for alkanes of the indicated chain lengths: $10<n<36$.

thickness is unaffected by the increased roughness. These results clearly demonstrate for C22 a stepwise, layer-by-layer growth of the Langmuir film, up to the completion of the third layer of flat lying molecules. Further increase in the coverage most probably results in the formation of 3D microcrystallites, which cause the roughening of the surface observed in the XR measurements. Towards the lower- $A$ end of the isotherm the microcrystallites become visible to the eye as nonreflecting white spots or lines on the shiny surface of the mercury.

These observations, and the average layer thickness of $4.6 \AA$ obtained from the fits, agree very well with the conclusions drawn above from the isotherms. This growth behavior is similar to that of SAMs of butane, hexane, and heptane on an $\operatorname{Ag}(111)$ surface at $40 \mathrm{~K}$, where these much shorter alkanes also form up to three molecular layers (depending on coverage) at the surface before bulk growth sets in. ${ }^{45}$ An important difference between that study and ours is, however, that the ordered Ag surface imposes long-range inplane order on the layers, as revealed by GID measurements. For the longer alkanes on mercury, studied here, no GID peaks are observed for any $n$, indicating that only shortrange in-plane order may exist, extending to a few molecular diameters only, as for a quasi-2D liquid or amorphous solid.

\section{B. Molecular length dependence}

\section{Surface pressure-molecular area isotherms}

The $\pi-A$ isotherms of alkanes on mercury are shown in Fig. 4 for several lengths $n$ at room temperature, $T$ $=23{ }^{\circ} \mathrm{C}$. Qualitatively, four types of isotherms are observed. Decane $(\mathrm{C} 10)$ and shorter alkanes exhibit a very low $\pi$ $(\$ 3-5 \mathrm{mN} / \mathrm{m})$ over most of the measurement range $18 \leqq A$ $\$ 400 \AA^{2} /$ molecule, without any prominent features, except for a small increase in $\pi$ towards the low- $A$ end of the isotherm. This increase (observed for $n \leqslant 14$ ) was found to depend on time rather than coverage, and probably results from the accumulation of contaminants on the not-fully-covered
TABLE I. The exclusion area $A_{1}$, and the onsets of the second and third plateaus $A_{2}$ and $A_{3} . \pi_{1}, \pi_{2}$, and $\pi_{3}$ are the surface pressures of the plateaus for the chain lengths, $n$, listed.

\begin{tabular}{|c|c|c|c|c|c|c|}
\hline \multirow[b]{2}{*}{$n$} & $A_{1}$ & $A_{2}$ & $A_{3}$ & $\pi_{1}$ & $\pi_{2}$ & $\pi_{3}$ \\
\hline & \multicolumn{3}{|c|}{$\AA^{2} / \mathrm{mol}$} & \multicolumn{3}{|c|}{$\mathrm{mN} / \mathrm{m}$} \\
\hline 10 & & & & 0 & & \\
\hline 11 & & & & 7 & & \\
\hline 12 & 87 & & & 13 & & \\
\hline 14 & 93 & & & 24 & & \\
\hline 16 & 109 & & & 34 & & \\
\hline 18 & 114 & & & 42 & & \\
\hline 19 & 122 & 60 & & 46 & 52 & \\
\hline 20 & 124 & 60 & 42 & 46 & 56 & 59 \\
\hline 21 & 131 & 63 & 44 & 46 & 56 & 59 \\
\hline 22 & 138 & 64 & 41 & 47 & 56 & 58 \\
\hline 24 & 152 & 70 & 48 & 44 & 54 & 56 \\
\hline 28 & 172 & 87 & & 42 & 54 & \\
\hline 36 & 233 & 105 & & 40 & 50 & \\
\hline 50 & 293 & 115 & & 40 & 51 & \\
\hline
\end{tabular}

high-energy surface of mercury, probably from the gas phase above, or even from the mercury bulk below the surface.

Decreasing the molecular area from $A$ $\approx 400 \AA^{2} /$ molecule a distinct steep rise in $\pi$ is observed for all $n \geqslant 11$, followed by a break and a plateau. The break occurs always at, or near, the molecular area of a flat-lying molecule, $A_{1}$, which increases linearly with $n$. The steeply rising part of all isotherms can be fit reasonably well by the Volmer equation, as shown for C22 in a dashed line in Fig. 2. We also observe a linear increase with $n$ in the surface pressure of the plateau for $11 \leqslant n \leqslant 20$. The isotherms for $11 \leqslant n$ $\leqslant 18$ show a single plateau. The isotherm of C19 shows two plateaus, while those of $20 \leqslant n \leqslant 24$ show three plateaus. For longer alkanes, $n \geqslant 28$, only two plateaus are observed, as found for $\mathrm{C} 19$. The area at the onset of the second $\left(A_{2}\right)$ and third $\left(A_{3}\right)$ plateaus are always equal to one-half and onethird of the area of a flat-lying single molecule, indicating that at these plateaus the film consists of two and three layers of flat-lying molecules. The areas and pressures obtained from the isotherms are summarized in Table I. Each entry is an average of values derived from three independent isotherms. The absolute surface pressures of the plateau varied by up to $5 \mathrm{mN} / \mathrm{m}$ between different experiments with the same material, while the difference in surface pressure between plateaus never varied by more than $1 \mathrm{mN} / \mathrm{m}$. The area per molecule at the onset of the first plateau varied between different isotherms by up to $5 \%$ when using the same solution, and up to $10 \%$, when using different solutions.

The exclusion area, $A_{1}$, obtained from the Volmer equation fit, and the onset areas, $A_{2}$ and $A_{3}$, are shown in Fig. 5, along with their linear fits. The larger error bars for $n=50$ reflect the difficulty in keeping these long molecules solvated, and the consequent larger scatter among the measured isotherms. All $A_{1}$ are very close to the expected area occupied by a single layer of closely packed, flat-lying molecules, and, as expected, increase linearly with $n$. The fit to a straight line yields $A_{1}=(6.2 \pm 0.2) n+(3 \pm 5) \AA^{2} /$ molecule. For the onsets of the second and third plateaus we obtain $A_{2}=(3.0 \pm 0.1) n \AA^{2} /$ molecule and $A_{3}=(2.0 \pm 0.1) n$ 


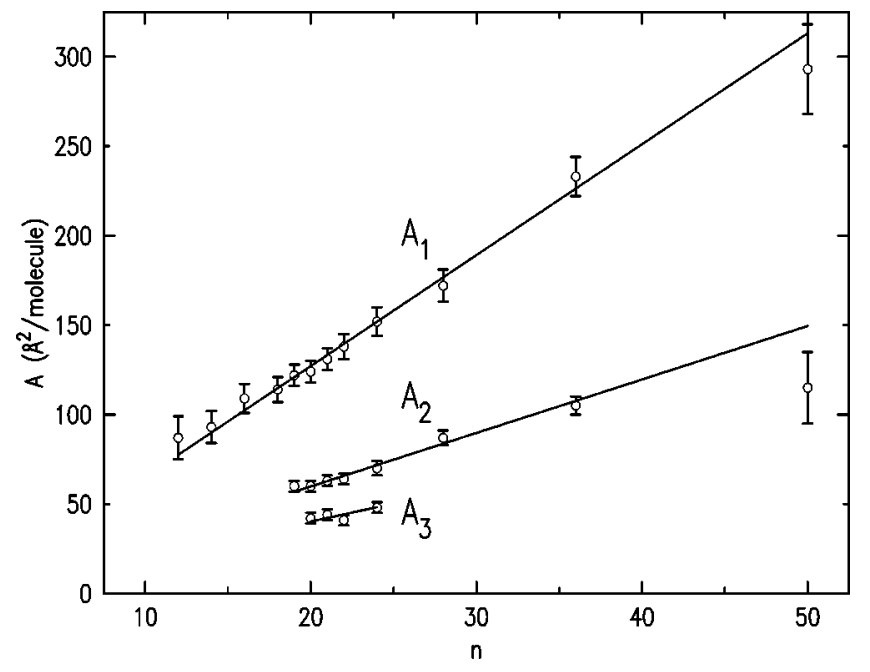

FIG. 5. Chain length dependence of the exclusion area $A_{1}$ obtained from the Volmer equation fit to the isotherms and the onsets of the second $\left(A_{2}\right)$ and third $A_{3}$ plateaus.

$\AA^{2} /$ molecule. The surface pressure at the onset of the first plateau increases linearly from $\pi \approx 0 \mathrm{mN} / \mathrm{m}$ for $n=10$ to $\pi$ $\approx 46 \mathrm{mN} / \mathrm{m}$ at $19 \leqslant n \leqslant 22$ then decreases again to $\pi$ $\approx 40 \mathrm{mN} / \mathrm{m}$ for $n \geqslant 36$. The surface pressure difference between the first and second plateaus is $\Delta \pi \approx 9-11 \mathrm{mN} / \mathrm{m}$ for all $n$ except for $n=19$, where $\Delta \pi \approx 6 \mathrm{mN} / \mathrm{m}$. The step from the second to the third plateau, where it exists, is $\Delta \pi$ $\approx 2-3 \mathrm{mN} / \mathrm{m}$. The implications of the $n$-variation of $A_{1,2,3}$ and $\pi_{1,2,3}$ are discussed below.

\section{X-ray reflectivity}

$\mathrm{X}$-ray reflectivity curves and corresponding electron density profiles, representative of the different chain length regimes discussed above, are shown in Fig. 6, for the alkanes and coverages indicated. We now discuss these in order of increasing $n$.
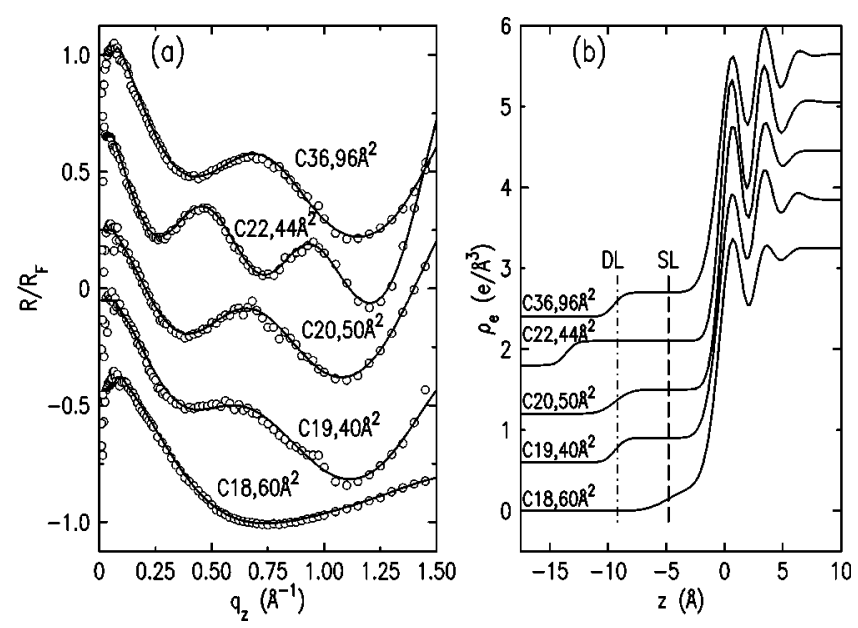

FIG. 6. (a) Measured Fresnel-normalized x-ray reflectivity curves (open circles) for the alkanes and coverages indicated, with their box-model fits (lines). Curves are shifted vertically by $\sim 0.3$ each for clarity. (b) The density profiles obtained from the fits, corresponding to the curves in (a). The vertical lines show the single (SL) and double (DL) layers.
The isotherms of C12-C16 in Fig. 4 show only a single plateau, pointing to the existence of a single flat-lying layer of alkanes. This is indeed found in the x-ray measurement. For C18, although the isotherm in Fig. 4 shows a small second step, the $\mathrm{x}$-ray measurements find just a single layer of flat-lying molecules with a thickness of $4.8 \AA$, as shown in Fig. 6 , and no other changes are observed upon varying $A$. The only change observed in the XR fits is an increase in the roughness, similar to that discussed above for $\mathrm{C} 22$, indicating the growth of three-dimensional crystallites.

The XR measurements of C19, however, show the formation of a double layer with a thickness of $9.4 \AA$ as expected from the isotherm, but no further increase in the layer thickness was observed with decreasing $A$. C20 also shows clearly a double layer $9.4 \AA$ thick. Both of these alkanes, show of course, in the x-ray measurements also a single layer, for lower coverages. Despite a weak third step in the isotherm of C20 no third layer could be observed in the XR measurements. This behavior can be explained by the growth of a metastable third layer with a lifetime long enough for measuring an isotherm but too short for measuring a reflectivity curve.

As discussed above, $\mathrm{C} 22$ shows, in addition to the single and double layer phases, a triple layer phase, the XR curve of which is shown in Fig. 6. This is the only chain length among those studied for which the triple layer phase was observed in the x-ray measurements. For longer chains the maximum number of layers found was two. A typical reflectivity for this regime of chain lengths is shown for C36 in Fig. 6, where the XR curve of the thickest film is that of a double layer phase. We now proceed to discuss the $(n, A, \pi)$ three-dimensional phase diagram emerging from the $\mathrm{x}$-ray and isotherm measurements.

\section{The phase diagram}

The isotherms and XR curves discussed above fully characterize the structure of the various phases of the mercury-supported Langmuir films of alkanes as a function of surface pressure $\pi$, area per molecule $A$ and chain length, $n$. The phase diagram is shown in Fig. 7. The gas phase of the flat-lying molecules is observed on the right-hand side, with a steep rise with decreasing $A$ to a condensed single layer phase. The onset of this condensed phase is characterized by a sharp bend of the isotherm from an steeply-rising to a horizontal curve. For $22 \leqslant n \leqslant 24$ we observe the formation of up to three layers of flat-lying molecules. Longer chain lengths, and slightly shorter ones $(n=19,20)$ show a maximum of two layers, and shorter chain lengths exhibit a single layer only. A projection of the phase diagram on the $\pi$ $n$ plane is shown in Fig. 8. It shows all the features described earlier, and the $n, \pi$-ranges of the gas, single, double, and triple condensed layer phases, up to $n=50$, the longest molecule investigated in this study. The collapse pressure, which is the equilibrium pressure between $2 \mathrm{D}$ and $3 \mathrm{D}$ growth, rises steeply for low chain lengths, reaches a maximum for $n$ $=22$, decreases slightly for $n>22$ and levels off for longer chain lengths. 


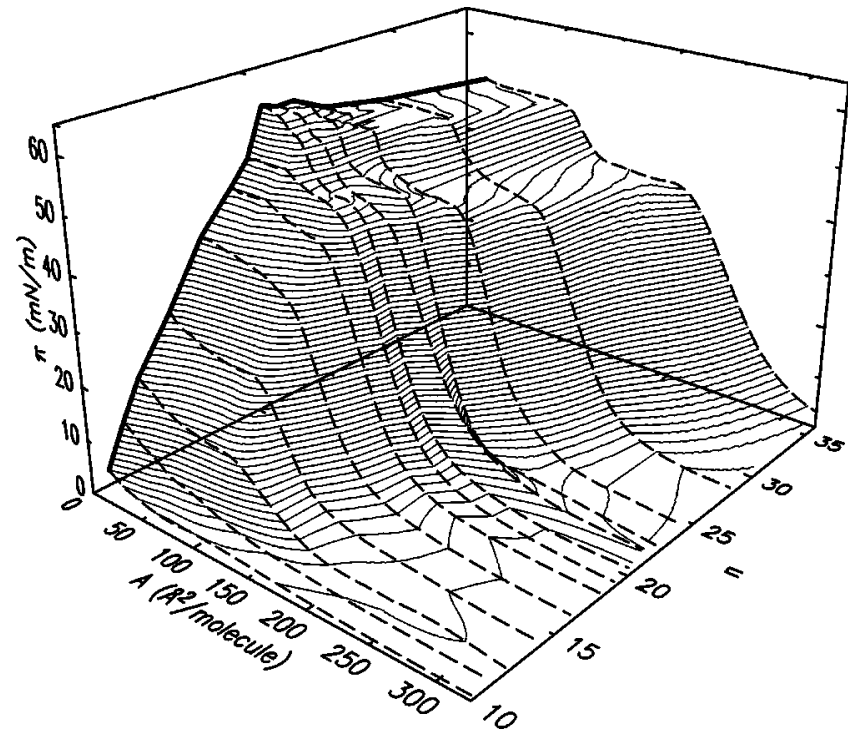

FIG. 7. The phase diagram of Langmuir films of alkanes on the surface of mercury. The measured isotherms are shown in a dashed line, and the collapse pressure—in a bold line.

\section{The collapse pressure and adsorption energy}

The collapse pressure of each isotherm and the maximal number of surface layers of the Langmuir film are determined by a balance between the energies of the $2 \mathrm{D}$ surface film and the 3D bulk. We note that at $T=23^{\circ} \mathrm{C}$, where our measurements were carried out, the bulk alkane is liquid for $n \leqslant 17$ and a solid for $n \geqslant 18$. Moreover, as Fig. 8 shows, $n$ $\approx 18$ is also the transition point below which only a single layer, and above which a double layer, are observed in the isotherm. For $n \leqslant 17$, where only a single 2D surface layer is observed, $A_{1}$ is the transition point between a $2 \mathrm{D}$ phase and a $3 \mathrm{D}$ one, i.e., the collapse point, since at this point the molecules of the 2D gas are maximally compressed, and are

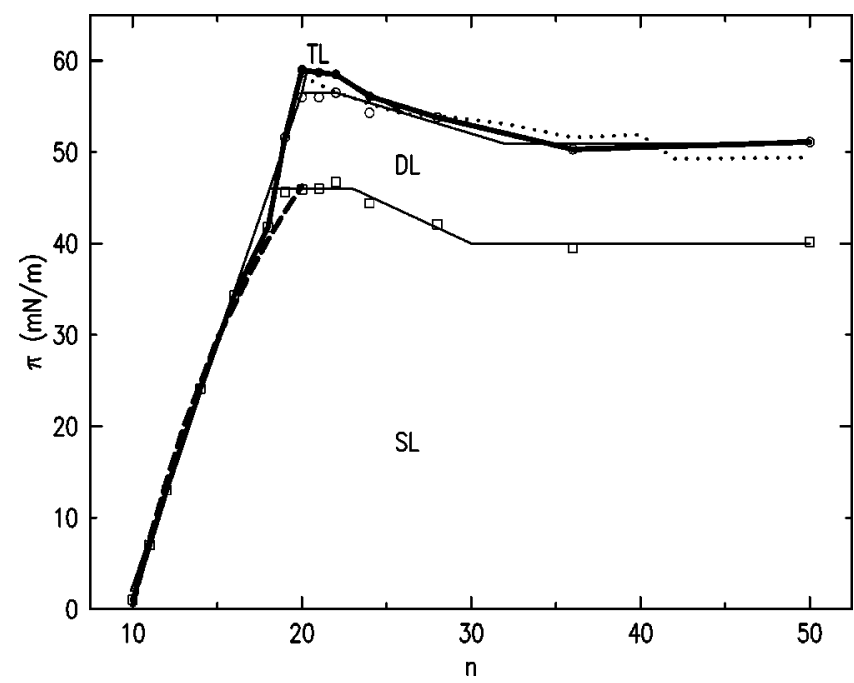

FIG. 8. $n-\pi$ phase diagram for alkanes adsorbed on mercury. The points are averages of the surface pressure at the phase transition between SL, DL, and TL for at least three different isotherms. The solid line are guides to the eye. The dashed and dotted lines are fits, discussed in the text, yielding the adsorption energies of the first and second condensed layers. The collapse pressure is shown as a bold line. touching each other. Any further reduction in the effective $A$ could come only by the expulsion of molecules from the surface layer into the $3 \mathrm{D}$ phase. Since for $n \leqslant 17$ the $3 \mathrm{D}$ phase is liquid, and for $A>A_{1}$ the $2 \mathrm{D}$ phase is a gas, the film collapse at $A_{1}$ can be considered as a condensation transition from a 2D-gas to a 3D-liquid. This transition is associated with a vaporization enthalpy which is derived below.

The same arguments show that for $n>18$, which exhibit a maximum of two surface layers (excluding $n=22$ where a TL phase was observed), the collapse point is at $A=A_{2}$, where the second layer is $100 \%$ complete, and any further reduction in $A$ must lead to the expulsion of molecules from the $2 \mathrm{D}$ surface phase into the $3 \mathrm{D}$ phase. The $3 \mathrm{D}$ phase here is known to be solid, but unlike the case for the 2D single layer case, where for $A>A_{1}$ the phase was shown to be a $2 \mathrm{D}$ gas, the exact nature of the $2 \mathrm{D}$ double layer phase at $A>A_{2}$ is not known. Since no GID peaks were observed, the DL phase is amorphous. As $A \ll A_{1}$ the DL phase is condensed, rather than a gas. Of the two remaining choices, i.e., a liquidlike or an amorphous-solidlike 2D phase, we have chosen the former. Thus, the collapse at $A_{2}$ can be considered to be a transition from a $2 \mathrm{D}$ liquid to a $3 \mathrm{D}$ solid, i.e., a freezing transition associated with a melting enthalpy.

As we show in the next paragraphs, and in Fig. 8, this general picture of the collapse agrees very well quantitatively with the measured collapse pressures, and their $n$ dependence.

To make the discussion above more quantitative we adopt a simplified view of the surface adsorption process of the alkane molecules as being controlled by a balance between two competing interactions and their associated energies. One is the attractive molecule-surface interaction which favors the formation of a $2 \mathrm{D}$ monolayer since the formation of such a layer lowers the total interfacial energy. This process is characterized by an adsorption energy. The other interaction is the molecule-molecule interaction which favors the formation of a 3D liquid or solid over the formation of a $2 \mathrm{D}$ layer. For $n$ up to $n \approx 18$ this interaction is characterized by the enthalpy of vaporization $\Delta H_{v}$. The adsorption energy of an alkane of $n$ carbons is $E_{\text {ads }}=(n$ -2) $\Delta E_{\mathrm{CH}_{2}}+2 \Delta E_{\mathrm{CH}_{3}}-E_{\text {defect }}$, where $\Delta E_{\mathrm{CH}_{2}}$ and $\Delta E_{\mathrm{CH}_{3}}$ are the adsorption energies of the $\mathrm{CH}_{2}$ and the $\mathrm{CH}_{3}$ groups, respectively. $E_{\text {defect }}$ is the reduction in the adsorption energy due to packing defects, caused, e.g., by gauche kinks which increase the average area per $\mathrm{CH}_{2}$ monomer over that of ideal packing. Since gauche kinks appear primarily at the ends of alkane chains and their number is mostly independent of the molecular length $n,{ }^{46} E_{\text {defect }}$ caused by gauche kinks should be independent of $n$, an assumption adopted in, and supported by, the fits discussed below. The surface pressure $\pi_{c}$ at collapse for chain lengths $n$ exhibiting a singlelayer plateau only, are then given by: $\pi_{c}=E_{\text {ads }}-\Delta H_{v}=(n$ -2) $\Delta E_{\mathrm{CH}_{2}}+2 \Delta E_{\mathrm{CH}_{3}}-E_{\text {defect }}-\Delta H_{v}$. Using published $\Delta H_{v}$ values $^{47,48}$ and assuming $\Delta E_{\mathrm{CH}_{2}} \approx \Delta E_{\mathrm{CH}_{3}}$, this expression can be fitted to the $\pi_{c}$ values derived from the measured isotherms, to obtain $\Delta E_{\mathrm{CH}_{2}}$ and $E_{\text {defect }}$. The fit, shown by a dashed line in Fig. 8, agrees very well with the experimental data for $n \lesssim 20$, even slightly higher than the expected $n$ 
$\lesssim 17$. It yields $\Delta E_{\mathrm{CH}_{2}}(\mathrm{SL})=5.4 \mathrm{~kJ} / \mathrm{mol}\left(\mathrm{CH}_{2}\right)$, close to the estimated adsorption enthalpy of alkyl chains on $\mathrm{Au}(111)$ of $6 \mathrm{~kJ} / \mathrm{mol}\left(\mathrm{CH}_{2}\right) .{ }^{49}$ We also obtain $E_{\text {defect }}=15 \mathrm{~kJ} / \mathrm{mol}$. This is about twice the $6.5 \mathrm{~kJ} / \mathrm{mol}$ energy cost of forming a gauche kink in an alkane chain. ${ }^{50}$ Moreover, the formation of a gauche kink in a molecule lying flat on the surface necessarily entails lifting part of the molecule off the surface, thereby causing a loss of the adsorption energy of that part. To a first approximation, assuming that a single $\mathrm{CH}_{2}$ group is lifted, the formation of a gauche kink costs 6.5+5.4 $=11.9 \mathrm{~kJ} / \mathrm{mol}$, close to the value found for $E_{\text {defect }}$. Thus, while a definite origin for $E_{\text {defect }}$, based on its numerical value, cannot be given here the comparison above demonstrates that its value as derived here, $E_{\text {defect }}=15 \mathrm{~kJ} / \mathrm{mol}$, is not unreasonable.

As discussed above, for longer chains, $n \geqslant 20$, the bulk phase is a solid at $T=23^{\circ} \mathrm{C}$, and the collapse is regarded as a $2 \mathrm{D}$-liquid to a $3 \mathrm{D}$-solid freezing transition. The collapse pressure is then determined by a balance between the adsorption energy and the enthalpy of melting: $\pi_{c}=E_{\mathrm{ads}}-\Delta H_{m}$. Using measured values of $\Delta H_{m}$ (Ref. 47) a fit to the measured collapse pressures, shown in a dotted line in Fig. 8, yields $\Delta E_{\mathrm{CH}_{2}}(\mathrm{DL})=9 \mathrm{~kJ} / \mathrm{mol}\left(\mathrm{CH}_{2}\right)$ for the double layer phase preceding collapse for $n \geqslant 20$. If the adsorption energy of the first, mercury-adjacent layer in this case is assumed to be unchanged from the $5.4 \mathrm{~kJ} / \mathrm{mol}\left(\mathrm{CH}_{2}\right)$ derived above from the single layer collapse pressure at $n \leqq 20$, the $\Delta E_{\mathrm{CH}_{2}}$ (DL) obtained indicates an adsorption energy of $3.6 \mathrm{~kJ} / \mathrm{mol}\left(\mathrm{CH}_{2}\right)$ for the second layer, two-thirds only of that of the first layer. A reliable calculation of the adsorption energy of the third layer directly from the triple layer phase's collapse pressures cannot be carried out because of the too-few data points available for the restricted $n$-range of this phase, as shown in Fig. 8. Finally, we point out that this phenomenological analysis may be oversimplified. A more sophisticated analysis, even at the mean-field Landau theory level akin to that carried out for fatty acids on water, ${ }^{3}$ is called for. This could perhaps be done when the temperature dependence of the phase diagram, now under study in our laboratory, is fully determined.

\section{Equilibrium versus nonequilibrium isotherms}

The general shapes of the isotherms measured here by stepwise deposition of molecules onto the surface are rather close to those measured by Smith $^{33}$ and Ellison, ${ }^{51}$ using compression of a fixed number of molecules by a traveling barrier. Close inspection, however, reveals a number of important differences. The number of plateaus obtained seems to be generally larger in the measurements of Smith and of Ellison than those observed here. For example, Smith observed three plateaus for $\mathrm{C} 32$ and two plateaus for both $\mathrm{C} 18$ and $\mathrm{C} 10$, while we observed two plateaus for $\mathrm{C} 28$ and $\mathrm{C} 36$, one plateau for $\mathrm{C} 18$ and no plateau for C10. Moreover, Smith's isotherms seem to reach higher surface pressures, and the plateaus have considerably larger slopes than the near-zero ones observed here. Smith's plateaus have also higher $A$-onsets than ours. As discussed in detail by Pershan et $a .^{52}$ for Langmuir films of behenic acid on water, these

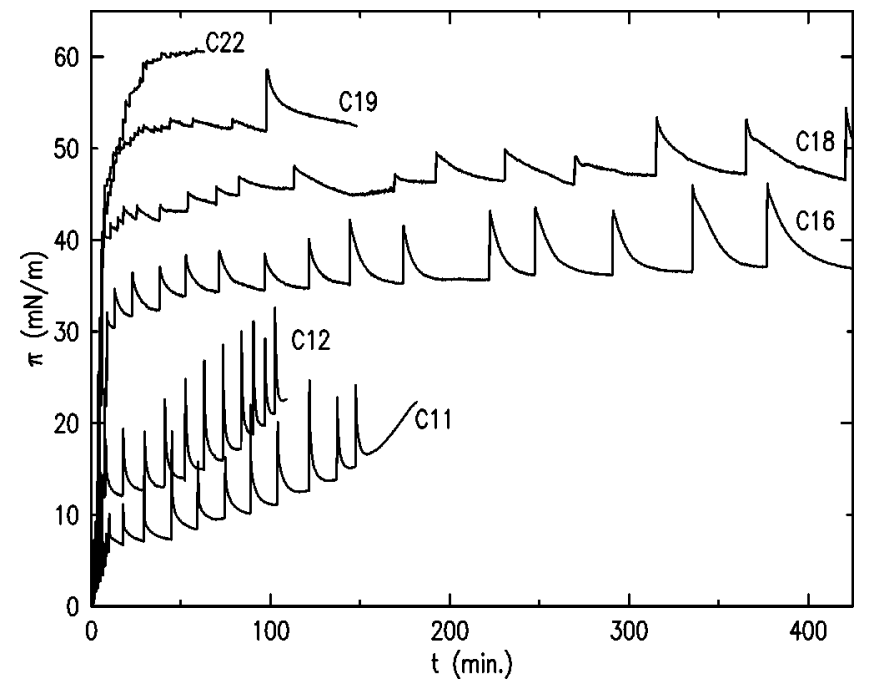

FIG. 9. Time evolution of the surface pressure during isotherm measurements, for different alkanes. The time to reach equilibrium pressure is very long ( $\$ 25 \mathrm{~min}$ ) for the high-coverage phases at medium-length alkanes $(\mathrm{C} 18, \mathrm{C} 19)$ and decrease for shorter as well as for longer alkanes.

symptoms suggest that Smith's isotherms, taken by rather fast barrier compression $\left(\sim 2.2 \mathrm{~cm}^{2} / \mathrm{s}\right)$, were most probably taken under nonequilibrium conditions, and are not, therefore, true thermodynamic equilibrium isotherms. As discussed above for $\mathrm{C} 22$, the method used here of adding material stepwise and waiting for the surface pressure to level out (see Fig. 1), most probably results in near-equilibrium conditions, as attested by the flat plateaus, lower onset $A \mathrm{~s}$ and sharper phase boundaries.

We now discuss some of the features observed in the time evolution of the surface pressure following a sample deposition step, and the variation of these features with chain length and number of surface layers. In Fig. 9 we show some of the pressure vs time plots recorded while measuring isotherms for alkanes of different lengths $n$. The typical jump in $\pi$ upon material addition, and the consequent relaxation of $\pi$, are clearly observed in each isotherm, and are generally similar to those shown in Fig. 1. For all alkanes with $n$ $\geqslant 20$ we observe very small decay times, $t \leqslant 1 \mathrm{~min}$, as discussed above for $\mathrm{C} 22$, over the full $A$ range of the isotherm, at all surface phases. The small decay times indicate a good spreading of the materials on the mercury surface and a high molecular mobility, even for double- or triple-layer phases. For these chain lengths, the rapid pressure relaxation allows completing the measurement of an isotherm with a sufficient number of points in about $1 \mathrm{~h}$.

For shorter chain lengths, a different behavior is observed. For C19 equilibrium pressure is reached rapidly for coverages $A$ down to the completion of the second layer. On further addition of material, 3D crystallites are eventually formed, and the relaxation times increases strongly to $t$ $\geqslant 15$ mins. The time dependence for $\mathrm{C} 18$ and $\mathrm{C} 16$ is similar. They show a strong increase in the relaxation times, up to $t$ $\geqslant 25$ mins, albeit this occurs here for a single layer, the only high-coverage surface phase observed for these $n$ (see Fig. 4). Both $\mathrm{C} 18$ and $\mathrm{C} 16$ show also a large jump upon addition of material, unlike those of longer alkanes, $n \geqslant 20$, which are 
relatively small. This may be indicative of an initial formation of a metastable double layer (which has a correspondingly higher $\pi$ ) which then transforms into the equilibrium state of 3D crystallites (C18) or droplets (C16) coexisting with a 2D single surface layer. In $\mathrm{C} 18$, in particular, the pressure relaxes very slowly at high coverages, and the relaxation curve is almost linear, indicating a very small gain in energy for the formation of $3 \mathrm{D}$ crystallite over the formation of a second 2D surface layer. A close look at C12 and C11 reveals an even larger initial jump in the surface pressure after deposition of new material. However, in contrast with $\mathrm{C} 16$ and $\mathrm{C} 18$, the pressure relaxes here exponentially to a constant value much more rapidly, $t \approx 2-3$ mins. This indicates a much faster growth of the $3 \mathrm{D}$ phase, which is not very surprising, considering that this phase is a liquid for these $n$. In fact, the fundamental reason for the large decrease in the relaxation times with $n$ from $\mathrm{C} 16$ to $\mathrm{C} 11$ is most probably the strong decrease in the viscosity, by a factor of 3 , with $n$ in this range, resulting in a much higher mobility of the molecules. This mobility enables a faster growth of the 3D liquid droplets, and thus a faster $\pi$ relaxation.

\section{CONCLUDING REMARKS}

The present x-ray and surface tension study of Langmuir films of alkanes on the surface of mercury demonstrates that for all alkanes studied $(10 \leqslant n \leqslant 50)$ the molecules are oriented parallel to the surface, in contrast with all Langmuir films of amphiphiles on aqueous subphases, where the molecular orientation is always along, or not excessively tilted away from the surface normal. The full $(n, A, \pi)$ phase diagram was determined, and exhibits a maximal number of three surface layers for $22 \leqslant n \leqslant 24$. For longer alkanes a maximum of two layers only are found. Shorter alkanes show a transition region, $n=19,20$ where a maximum of two layers are formed, while for $n<19$ only a single surface layer is observed. No long-range in-plane order is found for any of the surface phases and lengths $n$ studied. Considering the film collapse as a transition from a 2D gas to a 3D liquid $(n \leqslant 18)$ or from a $2 \mathrm{D}$ liquid to a $3 \mathrm{D}$ solid $(n \geqslant 20)$, allows deriving from the measured collapse pressures of the various $n$ the adsorption energy of a $\mathrm{CH}_{2}$ group at each layer. These considerations, as well as the observed $n$-variation of the surface pressure relaxation time following a sample deposition step, demonstrate the strong dependence of the Langmuir film's properties (maximal number of layers, plateau pressures, relaxation times, etc.) on the properties of the bulk phase at the measurement temperature. This connection is further investigated in a study, now in progress, of the temperature dependence of the structure of these films.

While no phases comprising surface-normal molecules are found here, fatty acids on mercury do show such phases. ${ }^{21,39}$ This fact highlights the importance of the head groups in determining the structure of Langmuir films on mercury. Another manifestation of the importance of the headgroup is the existence of long-range, smectic-like 1D long-range in-plane order in the flat-lying phases of $\mathrm{C} 18$ fatty acid, ${ }^{21}$ and 2D long-range in-plane order in C24 fatty acid Langmuir films on mercury. ${ }^{40}$ Studies of organic mol- ecules with different head groups should provide a deeper understanding of the role of the head group in inducing order in flat-lying and standing-up phases of Langmuir films of organic molecules on mercury. The thiol moiety is of particular interest in this respect, in view of the broad interest, and numerous studies, of various thiolates on single- and polycrystalline gold surfaces published over the last decade. ${ }^{26,53}$ Investigations of such compounds on mercury are currently in progress.

\section{ACKNOWLEDGMENTS}

Support to M.D. by the U.S.-Israel Binational Science Foundation, Jerusalem and to P.S.P. by the U.S. DOE (Grant No. DE-FG02-88-ER45379) and the NSF (Grant No. NSFDMR-0124936) is gratefully acknowledged. We thank D. Vaknin (Ames) for generous advice and drawings of the trough. BNL is supported by U.S. DOE Contract No. DEAC02-98CH10886.

${ }^{1}$ A. Pockels, Nature (London) 43, 437 (1891)

${ }^{2}$ Lord Rayleigh, Philos. Mag. 48, 321 (1899).

${ }^{3}$ V. M. Kaganer, H. Möhwald, and P. Dutta, Rev. Mod. Phys. 71, 779 (1999).

${ }^{4}$ S. Zheng, J. Strzalka, D.H. Jones, S.J. Opella, and J.K. Blasie, Biophys. J. 84, 2393 (2003); G. Brezesinski and H. Möhwald, Adv. Colloid Interface Sci. 100, 563 (2003).

${ }^{5}$ H. Rapaport, I. Kuzmenko, M. Berfeld, K. Kjaer, J. Als-Nielsen, R. Popovitz-Biro, I. Weissbuch, M. Lahav, and L. Leiserowitz, J. Phys. Chem. B 104, 1399 (2000).

${ }^{6}$ M. Gleiche, L.F. Chi, and H. Fuchs, Nature (London) 403, 173 (2000); A.L.Simões Gamboa, E.J.M. Filipe, and P. Brogueira, Nano Lett. 2, 1083 (2002); H. Lee, E. Bae, and W. Lee, Thin Solid Films 393, 237 (2001).

${ }^{7}$ Molecular Electronics, edited by J. Jortner and M. Ratner (Blackwell, Oxford, 1997); P. Samori and J.P. Rabe, J. Phys.: Condens. Matter 14, 9955 (2002).

${ }^{8}$ D.K. Schwartz, Surf. Sci. Rep. 27, 245 (1997).

${ }^{9}$ R.H. Tredgold, Order in Thin Organic Films (Cambridge University Press, Cambridge, 1994).

${ }^{10}$ G.L. Gaines, Insoluble Monolayers at Liquid-Gas Interfaces (Wiley, New York, 1966).

${ }^{11}$ J. Als-Nielsen, F. Christensen, and P.S. Pershan, Phys. Rev. Lett. 48, 1107 (1982); P.S. Pershan and J. Als-Nielsen, ibid. 52, 759 (1984).

12 J. Als-Nielsen, F. Christensen, and P.S. Pershan, Phys. Rev. Lett. 48, 1107 (1982).

${ }^{13}$ J. Als-Nielsen, D. Jacquemain, K. Kjaer, F. Leveiller, M. Lahav, and L. Leiserowitz, Phys. Rep. 246, 252 (1994).

${ }^{14}$ J. Penfold, Rep. Prog. Phys. 7, 777 (2001).

${ }^{15}$ A. Braslau, M. Deutsch, P.S. Pershan, A.H. Weiss, J. Als-Nielsen, and J. Bohr, Phys. Rev. Lett. 54, 114 (1985).

${ }^{16}$ S. Grayer Wolf, L. Leiserowitz, M. Lahav, M. Deutsch, K. Kjaer, and J. Als-Nielsen, Nature (London) 328, 63 (1987); K. Kjaer, J. Als-Nielsen, C.A. Helm, L.A. Laxhuber, and H. Möwald, Phys. Rev. Lett. 58, 2224 (1987); P. Dutta, J.B. Peng, B. Lin, J.B. Ketterson, M. Prakash, P. Georgopoulos, and S. Ehrlich, ibid. 58, 2228 (1987).

${ }^{17}$ I. Kuzmenko, H. Rapaport, K. Kjaer, J. Als-Nielsen, I. Weissbuch, M. Lahav, and L. Leiserowitz, Chem. Rev. 101, 1659 (2001).

${ }^{18}$ M. Fukuto, R.A. Heilmann, P.S. Pershan, S.M. Yu, C.M. Soto, and D.A. Tirrell, J. Chem. Phys. 119, 6253 (2003); M. Fukuto, R.A. Heilmann, P.S. Pershan, S.M. Yu, J.A. Griffiths, and D.A. Tirrell, J. Chem. Phys. 111, 9761 (1999); M. Fukuto, R.A. Heilmann, P.S. Pershan, S.M. Yu, C.M. Soto, and D.A. Tirrell, Phys. Rev. E 66, 010601(R) (2002); D. Vaknin, J.Y. Wang, and R.A. Uphaus, Langmuir 11, 1435 (1995); N.C. Maliszewskyj, P.A. Heiney, D.R. Jones, R.M. Strongin, M.A. Cichy, and A.B. Smith, ibid. 9, 1439 (1993).

${ }^{19}$ I.R. Peterson, V. Brezezinski, R.M. Kenn, and R. Steitz, Langmuir 8, 2995 (1992).

${ }^{20}$ S.P. Weinbach, K. Kjaer, J. Als-Nielsen, M. Lahav, and L. Leiserowitz, J, Phys. Chem. 97, 5200 (1993). 
${ }^{21}$ H. Kraack, B.M. Ocko, P.S. Pershan, E. Sloutskin, and M. Deutsch, Science 298, 1404 (2002).

${ }^{22}$ S.P. Weinbach, I. Weisbuch, K. Kjaer, W.G. Bowman, J. Als-Nielsen, M. Lahav, and L. Leiserowitz, Adv. Mater. (Weinheim, Ger.) 7, 857 (1995).

${ }^{23}$ O.M. Magnussen, B.M. Ocko, M.J. Regan, M.J. Penanen, M. Deutsch, and P.S. Pershan, Phys. Rev. Lett. 74, 4444 (1995); E. DiMasi, H. Tostmann, B.M. Ocko, P.S. Pershan, and M. Deutsch, Phys. Rev. B 58, R13419 (1998).

${ }^{24}$ O.M. Magnussen, B.M. Ocko, M. Deutsch, M.J. Regan, P.S. Pershan, D. Abernathy, G. Grübel, and J.F. Legrand, Nature (London) 384, 250 (1996).

${ }^{25}$ M. Deutsch, O.M. Magnussen, B.M. Ocko, M.J. Regan, and P.S. Pershan, in Thin Films: Self-Assembled Monolayers of Thiols, edited by A. Ulman (Academic, San Diego, 1998).

${ }^{26}$ F. Schreiber, Prog. Surf. Sci. 65, 151 (2000).

${ }^{27}$ L. Strong and G. Whitesides, Langmuir 4, 546 (1988); C.E.D. Chidsey and D.N. Loiacono, ibid. 6, 682 (1990).

${ }^{28}$ N. Camillone, P. Eisenberger, T.Y.B. Leung, P. Schwartz, G. Scoles, G.E. Poirier, and M.J. Tralov, J. Chem. Phys. 101, 11031 (1994).

${ }^{29}$ J.P. Rabe and S. Buchholz, Science 253, 424 (1991); T. Arnold, C.C. Dong, R.K. Thomas, M.A. Castro, A. Perdigon, S.M. Clarke, and A. Inaba, Phys. Chem. Chem. Phys. 4, 345 (2002).

${ }^{30}$ Y. Hosoi, Y. Hosoi, Y. Sakurai, M. Yamamoto, H. Ishii, Y. Ouchi, and K. Seki, Surf. Sci. 515, 157 (2002).

${ }^{31}$ A. Marchenko, J. Cousty, and L.P. Van, Langmuir 18, 1171 (2002).

${ }^{32}$ S.K. Lahiri and D. Gupta, J. Appl. Phys. 51, 5555 (1980).

${ }^{33}$ T. Smith, Adv. Colloid Interface Sci. 3, 161 (1972).

${ }^{34}$ S.W. Barton, B.N. Thomas, F. Novak, P.M. Weber, J. Harris, P. Dolmer, J.M. Bloch, and S.A. Rice, Nature (London) 321, 685 (1986); B.C. Lu and S.A. Rice, J. Chem. Phys. 68, 5558 (1978); L. Bosio and M. Oumezine, ibid. 80, 959 (1984); L. Bosio, R. Cortes, G. Folcher, and M. Froment, J. Electrochem. Soc. 139, 2110 (1992).

${ }^{35}$ M. Deutsch and B. M. Ocko, in Encyclopedia of Applied Physics, edited by G.L. Trigg (VCH, New York, 1998), Vol. 23, p. 479.

${ }^{36}$ T.R. Jensen and K. Kjaer, in Novel Methods to Study Interfacial Layers, edited by D. Möbius and R. Miller (Elsevier, Amsterdam, 2001).

${ }^{37}$ J. Lekner, Theory of Reflection (Martinus Nijhoff, Dordrecht, 1987); F. Abeles, Ann. Phys. (Paris) 5, 596 (1950); B.M. Ocko, X.Z. Wu, E.B.
Sirota, S.K. Sinha, O. Gang, and M. Deutsch, Phys. Rev. E 55, 3164 (1997).

${ }^{38}$ L.G. Parratt, Phys. Rev. 95, 359 (1954).

${ }^{39}$ H. Kraack, M. Deutsch, B.M. Ocko, and P.S. Pershan, Nucl. Instrum. Methods Phys. Res. B 200, 363 (2003).

${ }^{40}$ H. Kraack, B.M. Ocko, P.S. Pershan, E. Sloutskin, L. Tamam, and M. Deutsch (unpublished).

${ }^{41}$ J. Israelachvilli, Intermolecular and Surface Forces, 2nd ed. (Academic, San Jose, 1992), Sec. 6.3.

${ }^{42}$ J. Israelachvilli, Langmuir 10, 3774 (1994). See also the extensive recent work by V.B. Fainerman and D. Volhardt, J. Phys. Chem. B 107, 3098 (2003), in Ref. 44, and several references therein. Note that in spite of the graphic similarity with our isotherms, our $A_{1}$ does not correspond to $A_{c}$ in these studies, but rather to $A_{0}$ and $\omega$, respectively, i.e., the area occupied by a single molecule at the monolayer's most compressed state.

${ }^{43}$ Dr. L. Tonks (private communication), cited in I. Langmuir, J. Am. Chem. Soc. 54, 2798 (1932).

${ }^{44}$ D. Vollhardt and V. Fainerman, Colloids Surf., A 176, 117 (2001); I. Langmuir, J. Chem. Phys. 1, 756 (1933); J.T. Davies, Proc. R. Soc. London, Ser. A 208, 224 (1951); J.N. Phillips and E. Rideal, ibid. 232, 149 (1955).

${ }^{45}$ Z. Wu, S.N. Ehrlich, B. Matthies, K.W. Herwig, P.C. Dai, U.G. Volkmann, F.Y. Hansen, and H. Taub, Chem. Phys. Lett. 348, 168 (2001).

${ }^{46}$ R.G. Snyder, M. Maroncelli, H.L. Strauss, C.A. Elliger, D.G. Cameron, H.L. Casal, and H.H. Mantsch, J. Am. Chem. Soc. 105, 133 (1983).

${ }^{47}$ Nist Chemistry Webbook online: http://webbook.nist.gov/chemistry/

${ }^{48}$ M. Small, The Physical Chemistry of Lipids (Plenum, New York, 1986).

${ }^{49}$ D.J. Lavrich, S.M. Wetterer, S.L. Bernasek, and G. Scoles, J. Phys. Chem. B 102, 3456 (1998).

${ }^{50}$ M.B. Robertson, Polymer 42, 1261 (2001).

${ }^{51}$ A.H. Ellison, J. Phys. Chem. 66, 1867 (1962).

${ }^{52}$ M.L. Schlossman, D.K. Schwartz, P.S. Pershan, E.H. Kawamoto, G.J. Kellogg, and S. Lee, Phys. Rev. Lett. 66, 1599 (1991); D.K. Schwartz, M.L. Schlossman, and P.S. Pershan, J. Chem. Phys. 96, 2356 (1992); G.M. Bommarito, W.J. Foster, P.S. Pershan, and M.L. Schlossman, J. Phys. Chem. 105, 5265 (1996).

${ }^{53}$ Thin Films: Self-Assembled Monolayers of Thiols, edited by A. Ulman (Academic, San Diego, 1998). 